\title{
Introducing an Experimental Design Approach for Efficient Optimization of Chiral Derivatization Conditions for D- and L-Glyceric Acids
}

\author{
Takahiro Takayama, Hajime Mizuno, Toshimasa Toyo’oka, and Kenichiro Todoroki ${ }^{\dagger}$ \\ Laboratory of Analytical and Bio-Analytical Chemistry, School of Pharmaceutical Sciences, \\ University of Shizuoka, 52-1 Yada, Suruga, Shizuoka 422-8526, Japan
}

\begin{abstract}
A sensitive analytical method was developed for individual analyses of D- and L-glyceric acids by chiral derivatization - liquid chromatography-tandem mass spectrometry. To elucidate rapid and efficient optimization for derivatization we newly introduced a concept of design of experiments (DOE). The optimization of major 5 factors in the derivatization could be predicted with only 28 measurements. By applying DOE to optimization, the yields of desired derivatives increased five-fold against before optimization.
\end{abstract}

Keywords Design of experiment, chiral derivatization, D- and L-glyceric acid

(Received March 6, 2019; Accepted May 29, 2019; Advance Publication Released Online by J-STAGE June 7, 2019)

\section{Introduction}

Glyceric acid (GA), which is an $\alpha$-hydroxylic acid metabolite, is serum and urine biomarkers increased in metabolic disorders. ${ }^{1-4}$ D- and L-chiral isomers of GAs are produced by D-GA kinase ${ }^{5,6}$ and dehydrogenase ${ }^{7,8}$ individually, which relate to different metabolic diseases. Thus, careful monitoring of GA isomers as biomarkers is very important for appropriate treatment.

We previously developed a chiral fluorescence derivatization - liquid chromatography (LC) method for D- and L-lactic acids (LAs) that uses 4-(4,6-dimethoxy-1,3,5-triazin-2-yl)-4-methylmorpholinium chloride (DMT-MM) as not only an amide formation agent, but also an enantioseparation enhancer. ${ }^{9,10}$ In this derivatization, the dimethoxytriazine (DMT) moiety of DMT-MM etherified the hydroxyl group of the D- and L-LAs derivative to produce a sterically bulky derivative. The resulting DMT introduced derivatives of D- and L-LA could be discriminated completely, and successfully enantioseparated through reversed-phase LC. Since this etherification of the DMT moiety is specific to $\alpha$-hydroxylic acids, we applied it to chiral derivatization - LC-tandem mass spectrometry (MS/ MS) analysis of D- and L-GAs. However, the reaction yield of the DMT introduction is not necessarily high. Therefore, optimization of the reaction conditions to obtain higher detection sensitivity and good precision has been required. The main factors affecting the derivatization reaction yield include the reaction time, reaction temperature, contents of a base catalyst and an organic solvent, and the concentration of reagents; it takes many experiments and much time to optimize these factors individually.

In this study, we newly introduced a concept of design of experiments (DOE) to elucidate rapid and efficient optimization

† To whom correspondence should be addressed.

E-mail: todoroki@u-shizuoka-ken.ac.jp for the chiral derivatization of $\mathrm{D}-$ and L-GAs. The DOE is a statistical method aimed at designing an efficient experimental method and analyzing the result appropriately. DOE can reasonably reduce repetitive experiments and combination experiments of various factors; therefore, it has been used for the optimization of LC separation, an establishment of quality by design in pharmaceutical development. ${ }^{11-15}$ As a chiralderivatization reagent, we selected $(S)$-1-(4,6-dimethoxy-1,3,5triazin-2-yl) pyrrolidin-3-amine (DMT- $(S)-\mathrm{A})$, which enables highly sensitive detection of chiral carboxylic acids with ESIMS/MS detection, ${ }^{16}$ and its derivatization conditions were optimized by DOE. This reaction scheme is shown in Fig. 1. Applications of DOE in optimizing the derivatization method has been limited to GC-MS analysis..$^{17,18}$ The present study is the first application for chiral derivatization — LC-MS/MS analysis.

\section{Experimental}

Reagents and chemicals

DL-GA (racemate) was purchased from Tokyo Chemical Industry (Tokyo, Japan). D-GA was also purchased from SigmaAldrich (St. Louis, MO). DMT-MM was obtained from Wako Pure Chemical Industries (Osaka, Japan). Acetonitrile $\left(\mathrm{CH}_{3} \mathrm{CN}\right)$, methanol $\left(\mathrm{CH}_{3} \mathrm{OH}\right)$, pyridine, triethylamine (TEA), and LC-MS grade of formic acid (FA) were purchased from Kanto Chemicals (Tokyo, Japan). Pooled human sera were obtained from Nissui Pharmaceutical (Tokyo, Japan). Water $\left(\mathrm{H}_{2} \mathrm{O}\right)$ was purified using the PURELAB flex 3 ultrapure water purification system (ELGA LabWater, High Wycombe, UK). Each $10 \mathrm{mmol} / \mathrm{L}$ stock solution of D- and DL-DA was prepared with $\mathrm{H}_{2} \mathrm{O}$ and sequentially diluted with $\mathrm{CH}_{3} \mathrm{CN}$ just before use. Synthesis of DMT- $(S)$-A was performed according to our previous method. ${ }^{16}$

\section{Apparatus}

An ultra-performance LC-electrospray spray ionization tandem mass spectrometry (UPLC-ESI-MS/MS) was performed 


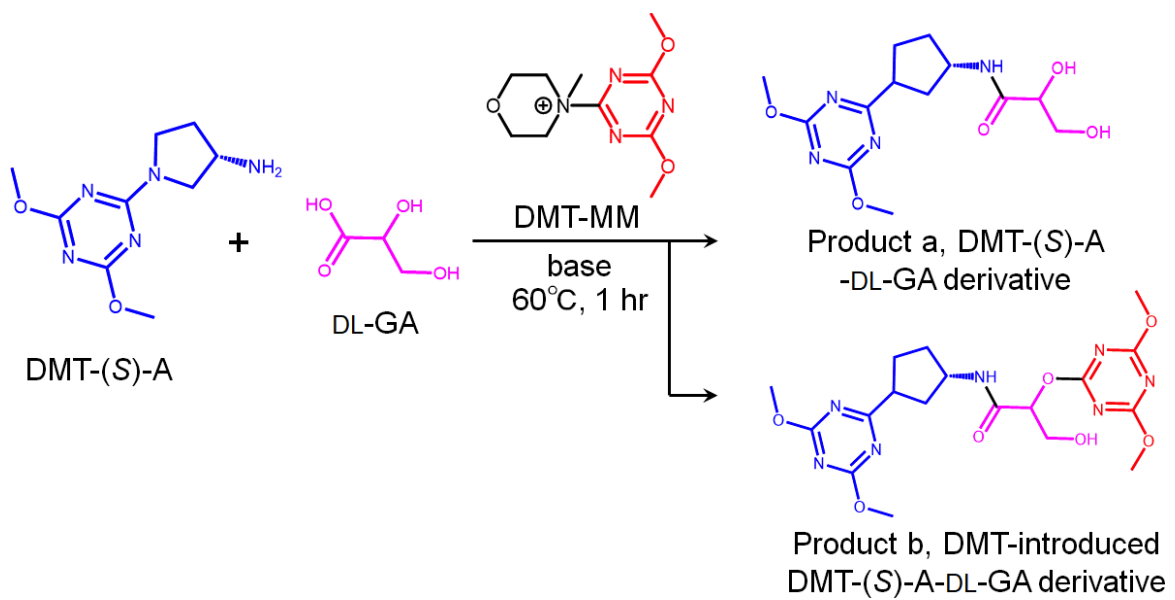

Fig. 1 Chiral derivatization for D- and L-GAs that uses DMT- $(S)$-A and DMT-MM. Product a, DMT$(S)$-A-DL-GA derivatives not introduced DMT moiety; b, DMT- $(S)$-A-DL-GA derivatives introduced DMT moiety

using an ACQUITY UPLC I-class (Waters, Milford, MA) connected to a Xevo TQS mass spectrometer (Waters). For MS detection of derivatives, the ESI positive mode and multiple reaction monitoring (MRM) mode were used. The analytical column used was an ACQUITY UPLC BEH C18 column $(1.7 \mu \mathrm{m}, 150 \times 2.1 \mathrm{~mm}$ i.d., Waters $)$. The elution conditions were as follows: mobile phase, $\mathrm{CH}_{3} \mathrm{CN} / \mathrm{H}_{2} \mathrm{O}$ mixture containing $0.1 \%$ (v/v) FA; isocratic elution $\% \mathrm{CH}_{3} \mathrm{CN}(\mathrm{min}), 12(0-20)-$ 95(21-22)-12(22.5-25). The flow rate of the mobile phase was $0.4 \mathrm{~mL} / \mathrm{min}$. The MS/MS conditions are detailed in Supporting Information.

\section{JMP software data analysis condition for DOE}

The JMP software (Ver. 13.0.0, SAS Institute Japan, Tokyo, Japan) was used to designing the experiment. The custom plan was applied to control the factors range and regulation, kind of plan, and criteria of optimization. The response surface design was selected to create an experimental table based on the central composed design, and the resulting design was evaluated by D-optimization criteria in this study. The variable of factors included squared, and entanglement term to observe the effect of the interaction. The response surface design by the central complex design, which was applied in this research, is one of method of DOE, which can evaluate the curved surface of a variation factor to a response factor. In 3-level analysis, central points and star points of each level of a factor are complementary employed while avoiding reduplication (Table 1). The defined conditions were actually analyzed, and the resulting peak areas in each condition were applied statistical modeling as described in below. A statistical prediction was performed by multipleregression modeling with a least-squares method. The modeling equation form was as follows;

$$
\begin{aligned}
Y= & A_{11} X_{1}^{2}+A_{22} X_{2}^{2}+A_{33} X_{3}^{2}+A_{44} X_{4}^{2}+A_{55} X_{5}^{2}+A_{12} X_{1} X_{2}+ \\
& A_{13} X_{1} X_{3}+A_{14} X_{1} X_{4}+A_{15} X_{1} X_{5}+A_{23} X_{2} X_{3}+A_{24} X_{2} X_{4}+ \\
& A_{25} X_{2} X_{5}+A_{34} X_{3} X_{4}+A_{35} X_{3} X_{5}+A_{45} X_{4} X_{5}+A_{1} X_{1}+ \\
& A_{2} X_{2}+A_{3} X_{3}+A_{4} X_{4}+A_{5} X_{5}+\varepsilon
\end{aligned}
$$

Here, $\mathrm{Y}$ is the response as peak area ratio of a sum of both DL-DA DMT introduced derivatives to DMT not introduced derivatives. $\mathrm{X}_{1 \sim \mathrm{n}}$ are variables including reaction temperature (RT), time, catalyst base addition amount (CB), DMT-MM concentration $(\mathrm{CD}), \mathrm{CH}_{3} \mathrm{CN}$ ratio in the reaction solution
Table 1 Experimental table provided by JMP software

\begin{tabular}{rcclrc}
\hline No & $\mathrm{RT} /{ }^{\circ} \mathrm{C}$ & Time/min & $\mathrm{CB}, \%$ & $\mathrm{CD} / \mathrm{mmol} \mathrm{L}^{-1}$ & $\mathrm{CH}_{3} \mathrm{CN}, \%$ \\
\hline 1 & 25 & 60 & 0 & 60 & 78 \\
2 & 25 & 10 & 0.5 & 60 & 78 \\
3 & 42.5 & 35 & 0.5 & 300 & 67 \\
4 & 60 & 10 & 0 & 300 & 56 \\
5 & 25 & 10 & 0 & 300 & 78 \\
6 & 25 & 60 & 0.5 & 60 & 67 \\
7 & 25 & 60 & 0 & 300 & 56 \\
8 & 60 & 35 & 0 & 300 & 78 \\
9 & 25 & 10 & 0.5 & 60 & 56 \\
10 & 60 & 60 & 0 & 60 & 56 \\
11 & 60 & 10 & 0.5 & 180 & 56 \\
12 & 60 & 60 & 0.5 & 60 & 78 \\
13 & 25 & 35 & 0 & 60 & 56 \\
14 & 42.5 & 60 & 0 & 300 & 78 \\
15 & 60 & 10 & 0.5 & 300 & 78 \\
16 & 60 & 10 & 0.25 & 60 & 67 \\
17 & 60 & 60 & 0.25 & 300 & 78 \\
18 & 42.5 & 60 & 0.25 & 60 & 56 \\
19 & 60 & 10 & 0 & 60 & 78 \\
20 & 25 & 60 & 0.5 & 180 & 56 \\
21 & 60 & 35 & 0.5 & 60 & 56 \\
22 & 42.5 & 10 & 0 & 180 & 56 \\
23 & 60 & 60 & 0.5 & 300 & 56 \\
24 & 25 & 60 & 0.5 & 300 & 78 \\
25 & 25 & 10 & 0 & 60 & 67 \\
26 & 25 & 10 & 0.5 & 300 & 56 \\
27 & 60 & 60 & 0 & 180 & 67 \\
28 & 25 & 35 & 0.25 & 180 & 78 \\
\hline
\end{tabular}

$\left(\mathrm{CH}_{3} \mathrm{CN}, \%\right) . \mathrm{A}_{1 \sim \mathrm{n}}$ are coefficients of $\mathrm{X}_{1 \sim \mathrm{n}}$ calculated from leastsquares modeling method. $\varepsilon$ is the error of the modeling method as the intercept of the function. In addition, to observe the interaction of each variable, and to perform the surface design, the interaction terms and square terms of each variable $\left(\mathrm{A}_{1 \sim \mathrm{n} 1 \sim \mathrm{n}}\right)$ were included in the model.

\section{Derivatization condition of $G A$}

An aliquot $(20 \mu \mathrm{L})$ of a sample solution containing DL-GA or D-GA was added to $20 \mu \mathrm{L}$ of $30 \mathrm{mmol} / \mathrm{L}$ DMT- $(S)-\mathrm{A}$ in $\mathrm{H}_{2} \mathrm{O} /$ $\mathrm{CH}_{3} \mathrm{CN}(1 / 1 \mathrm{v} / \mathrm{v})$ containing $0.5 \%$ TEA, $10 \mu \mathrm{L}$ of $300 \mathrm{mmol} / \mathrm{L}$ 
A

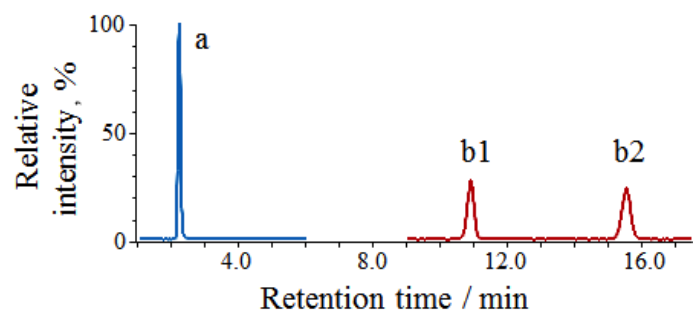

B

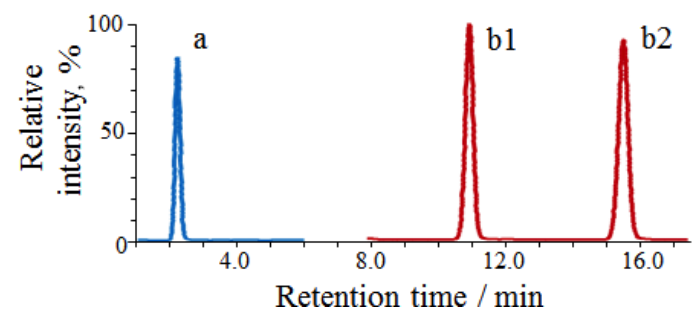

Fig. 2 MRM chromatograms of $20 \mu \mathrm{mol} / \mathrm{L}$ DL-GAs derivatized with DMT- $(S)$-A under (A) the optimized conditions for D- and L-LAs ${ }^{9}$ and (B) after optimization by the DOE. The peaks signed a, DMT not introduced derivatives of DL-GAs; b1, DMT introduced derivatives of D-GA; b2, DMT introduced derivatives of L-GA.

DMT-MM in $\mathrm{H}_{2} \mathrm{O} / \mathrm{CH}_{3} \mathrm{CN}(1 / 1 \mathrm{v} / \mathrm{v})$. This working solution was added to $50 \mu \mathrm{L} \mathrm{CH}_{3} \mathrm{CN}$ containing $0.2 \%$ TEA in order to control the concentration of TEA and the percentage of $\mathrm{CH}_{3} \mathrm{CN}$. This solution was stored at $60^{\circ} \mathrm{C}$ for $1 \mathrm{~h}$, and then added to $100 \mu \mathrm{L}$ of $0.1 \%$ FA in $\mathrm{CH}_{3} \mathrm{CN} / \mathrm{MeOH} / \mathrm{H}_{2} \mathrm{O}(1 / 1 / 3, \mathrm{v} / \mathrm{v} / \mathrm{v})$. When performing DOE optimization, the reaction temperature (RT), reaction time, catalyst base addition amount (CB), DMT-MM concentration (CD), percentage of $\mathrm{CH}_{3} \mathrm{CN}$ ratio in the reaction solution $\left(\mathrm{CH}_{3} \mathrm{CN}\right)$ were set as described in Table 1 .

Validation test of D- and L-GAs quantification analysis on serum A pooled human serum sample $(100 \mu \mathrm{L})$ was deproteinized by adding it to $1.9 \mathrm{~mL}$ of $\mathrm{CH}_{3} \mathrm{CN}$. After letting stand at room temperature for 15 -min, the mixture was centrifuged at $3000 \times g$ for $10 \mathrm{~min}$. The supernatant $(20 \mu \mathrm{L})$ was added to $50 \mu \mathrm{L}$ of different concentrations $(0-20 \mu \mathrm{M})$ of DL-GA diluted in $\mathrm{CH}_{3} \mathrm{CN}$ containing $0.2 \%$ TEA. These mixtures were applied to the derivatization procedure. Calibration curves were constructed by plotting the peak area versus the subjected amounts (50, 100, 200, 400, 800, 3000, $5000 \mathrm{fmol}$ on column). An recovery test from serum was performed at two concentrations (500 and $1500 \mathrm{fmol}$ on the column). Intra- and inter-day precisions were performed by quantifying DL-GAs existing in pooled human sera in 3 days $(n=3$ each day).

\section{Results and Discussion}

Figure 2A shows a typical MRM chromatogram of $20 \mu \mathrm{mol} / \mathrm{L}$ DL-GA derivatized with DMT- $(S)$-A under previously optimized conditions for D- and L-LAs. ${ }^{9}$ The retention times of the DMT introduced derivatives of D- and L-GA were 11 and $15.5 \mathrm{~min}$, respectively, with a peak resolution $\left(R_{\mathrm{s}}\right)$ of 2.86 . In contrast, the diastereoisomeric derivatives that were not modified with a DMT moiety were detected near $2.5 \mathrm{~min}$ as a single and large peak. The peak area ratio of the sum of both DMT introduced derivatives to DMT not introduced derivatives $((\mathrm{b} 1+\mathrm{b} 2) / \mathrm{a})$ was
A

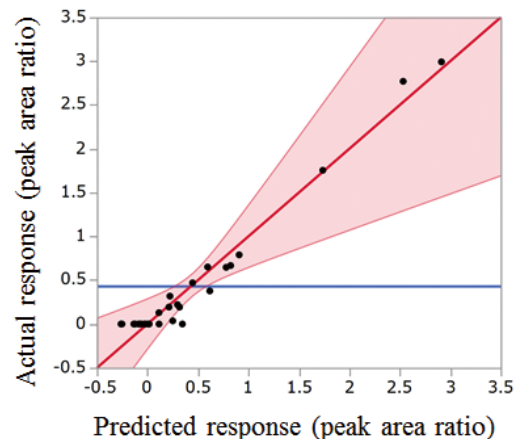

B

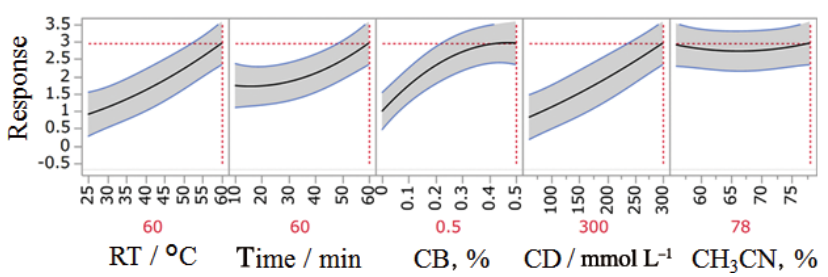

Fig. 3 (A) Accuracy of the statistical modeling obtained from DOE for optimization of the DMT-introduction reaction. (B) Determination of the optimization reaction conditions. The gray area in each graph of the factor indicates confidence interval. Response in each vertical of the figure is the peak-area ratio calculated by the following equation: peak area of $(b 1+b 2) / a$ denoted in Fig. 2. The red in the Fig. 3(B) represents the derived optimal values.

0.64. We carried out DOE to concisely determine the optimized derivatization conditions. As shown in Table 1, we investigated 5 factors, i.e., $\mathrm{RT}$, reaction time, $\mathrm{CB}, \mathrm{CD}, \mathrm{CH}_{3} \mathrm{CN}$, at 3 levels each. In a full factorial design that analyzes all combinations, $3^{5}=243$ experiments need to be considered. In contrast, a custom plan according to the central composite design could reduce the number of experiments to 28 times. DL-GA samples were derivatized with these 28 conditions, and then were analyzed by LC-MS/MS to obtain their peak-area ratios for use as response variables in the statistical prediction model. Figure 3A graphs an estrangement between the actual and predicted response. A good linearity $\left(R^{2}=0.91\right)$ was obtained between the prediction and the actual measurement; the root square mean error (RSME) was 0.263 , which is about an $8 \%$ error of the vertical axis maximum value. These results suggested that an accurate prediction of the model could be obtained by this DOE. Figure 4 shows the results of the significance test on each factor by the likelihood ratio test. Since the significant contribution $(p<0.01)$ was observed in $\mathrm{CB}, \mathrm{RT}, \mathrm{CD}$ and these combinations, these three factors particularly influenced the derivatization yield. From the prediction profile curves for each factor, $\mathrm{RT}=60^{\circ} \mathrm{C}$, time $=$ $60 \mathrm{~min}, \mathrm{CB}=0.5 \%, \mathrm{CD}=300 \mathrm{mmol} / \mathrm{L}, \mathrm{CH}_{3} \mathrm{CN}=78 \%$ were calculated as the optimum, respectively (Fig. 3B). Under these conditions, the peak area of DMT introduced derivatives was greatly increased by about 5 fold compared to those given by the initial conditions (Fig. 2B), and peak-area ratio was 2.96 . We also validated for D- and L-GAs spiked with pooled human sera as a biological specimen (Fig. S1 and Table S1, Supporting Information). No difference of slopes in the calibration curves was observed between the DL-isomers with good linearity $\left(r^{2}>0.997\right)$. The recovery rates at 500 and $1500 \mathrm{fmol}$ on the column were $91.3-110.1 \%$, intra-day $(n=3)$ and inter-day 


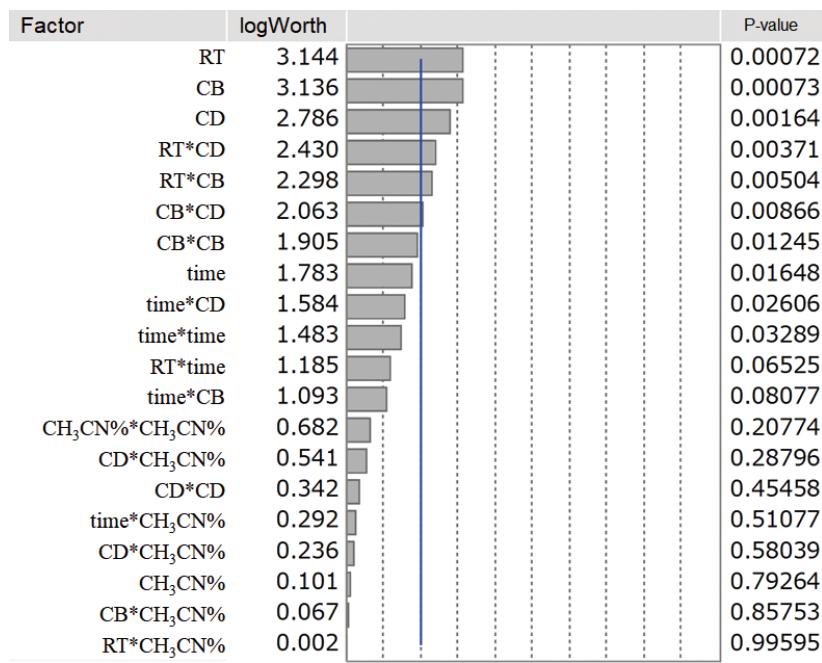

Fig. 4 Significance test of contribution of each factor to the response variation. The $p$-values were calculated based on the likelihood ratio test.

$(n=3)$ precisions were all within $10 \%$. The LOD and the LOQ were 11 and $33 \mathrm{fmol}$ on the column. The derivatization yields of DMT introduced derivatives could not be reached at $100 \%$, and they satisfied sufficient sensitivity to quantify D- and L-GAs in biological samples. ${ }^{2,3,8,9}$

\section{Conclusions}

In this study, we newly introduced the concept of DOE to elucidate rapid and efficient optimization for the chiral derivatization of $\mathrm{D}-$ and L-GAs. The optimization of major 5 factors in the derivatization could be predicted with only 28 measurements. By applying the DOE to the optimization, the yields of DMT introduced derivatives increased by five-fold against before optimization. From the results of the analytical validation that uses human serum samples, constructed a bioanalytical method for $\mathrm{D}$ - and L-GAs is applicable to the individual quantification of isomers in human body fluids; it would be useful for early diagnosis and discrimination of therapeutics effect in disease associated with functions of D-GA kinase and D-GA dehydrogenase. Our recent findings showed that D- and L-GAs are potential chiral diagnostic markers contained in the cerebrospinal fluid of patients with Alzheimer's disease; ${ }^{19}$ our method would also be applicable for this further investigation.

The proposed methodology is applicable to not only similar derivatization-LC-MS/MS analyses for other chiral $\alpha$-hydroxylic acids, but also other derivatization analyses not using DMTMM.

\section{Acknowledgements}

This study was supported by a Grant-in-Aid for JSPS Research
Fellow (No. 16J11918) from Japan Society for the Promotion of Science (JSPS).

\section{Supporting Information}

Additional details of the experiments, one table; result of validation test in human sera, and one figure; calibration curve of DL-GA spiked in human sera. These materials are available free of charge on the Web at http://www.jsac.or.jp/analsci/.

\section{References}

1. N. W. Dimer, P. F. Schuck, E. L. Streck, and G. C. Ferreira, An. Acad. Bras. Cienc., 2015, 87, 1409.

2. D. J. Dietzen, T. R. Wilhite, D. N. Kenagy, D. S. Milliner, C. H. Smith, and M. Landt, Clin. Chem., 1997, 43, 1315.

3. A. Kaunzinger, A. Rechner, T. Beck, A. Mosandl, A. C. Sewell, and H. Böhles, Enantiomer, 1996, 1, 177.

4. B. Hoppe and C. B. Langman, Pediatr. Nephrol., 2003, 18, 986.

5. J. R. Bonham, T. J. Stephenson, K. H. Carpenter, J. M. Rattenbury, C. H. Cromby, R. J. Pollitt, and D. Hull, Pediatr. Res., 1990, 28, 38.

6. G. E. Frauendienst and K. T. Friedrich, MetaGene: Metabolic \& Genetic Information Center (MIC: http:// www.metagene.de), METAGENE consortium, 2017.

7. M. S. Rashed, H. Y. Aboul-Enein, M. AlAmoudi, M. Jakob, L. Y. Al-Ahaideb, A. Abbad, S. Shabib, and E. Al-Jishi, Biomed. Chromatogr., 2002, 16, 191.

8. Y. Inoue, T. Shinka, M. Ohse, and T. Kuhara, J. Chromatogr. $B, \mathbf{2 0 0 5}, 1,2$.

9. K. Todoroki, K. Goto, T. Nakano, Y. Ishii, J. Z. Min, K. Inoue, and T. Toyo' oka, J. Chromatogr. A, 2014, 1360, 188.

10. K. Todoroki, M. Nakamura, Y. Sato, K. Goto, T. Nakano, Y. Ishii, J. Z. Min, K. Inoue, and T. Toyo'oka, Chromatography, 2016, 37, 23.

11. A. Desai, N. Raotole, G. Samanta, N. Kadam, and T. Tol, J. Chromatogr. A, 2016, 1432, 26.

12. T. Kawabe, T. Tomitsuka, T. Kajiro, N. Kishi, and T. Toyo'oka, J. Chromatogr. A, 2013, 1273, 95.

13. M. Taraji, P. R. Haddad, R. I. J. Amos, M. Talebi, R. Szucs, J. W. Dolan, and C. A. Pohl, Anal. Chem., 2017, 89, 1870.

14. T. Sasaki, K. Todoroki, and T. Toyo'oka, J. Pharm. Biomed. Anal., 2017, 146, 251.

15. P. Li, W. Sun, L. Zuo, Z. Zhu, T. Zhao, R. Wang, M. Zhu, X. Leng, X. Qiu, and L. Bai, J. Pharm. Biomed. Anal., 2018, 161, 407.

16. T. Takayama, T. Kuwabara, T. Maeda, I. Noge, Y. Kitagawa, K. Inoue, K. Todoroki, J. Z. Min, and T. Toyo'oka, Anal. Bioanal. Chem., 2015, 407, 1003.

17. A. Manca, E. Alladio, P. Massarenti, M. P. Puccinelli, A. De Francesco, E. Del Grosso, G. Mengozzi, M. Pazzi, and M. Vincenti, J. Chromatogr. B, 2017, 1070, 15.

18. T. Hložek, M. Bursová, P. Coufal, and R. Čabala, J. Anal. Toxicol., 2016, 40, 749.

19. T. Takayama, H. Mizuno, T. Toyo’oka, H. Akatsu, K. Inoue, and K. Todoroki, Anal. Chem., 2019, 91, 43396. 